\section{Escala de Depressão Pós-Parto de Edimburg: análise fatorial e desenvolvimento de uma versão de seis itens}

\section{Edimburg Postpartum Depression Scale: factorial analyses and development of six items version}

\section{Caro Editor,}

O período da gestação e do pós-parto implicam em um elevado risco para a mulher no que diz respeito ao desenvolvimento de psicopatologias. A prevalência da depressão pós-parto (DPP) é elevada, sendo que no Brasil os resultados variam entre $12 \mathrm{e}$ $39,4 \%$ das mulheres após o parto ${ }^{1}$. As causas da DPP envolvem fatores biológicos e sociais ${ }^{2}$. Além de ser um quadro altamente prejudicial para a gestante e seus familiares, a DPP geralmente é acompanhada de outros quadros comórbidos, o que aumenta a gravidade desta condição ${ }^{3}$. Apesar da existência de diversos estudos sobre aspectos epidemiológicos e clínicos sobre a DPP, em muitas situações o diagnóstico não é feito de forma precoce e adequada devido a questôes culturais (ex.: a mulher minimiza os sintomas sentindo-se culpada pelo humor depressivo após o parto), metodológicas (ex.: falta de instrumentos com boas propriedades psicométricas e de critérios objetivos para o diagnóstico) e pela própria heterogeneidade das manifestaçōes clínicas da DPP.

A Escala de Depressão Pós-Parto de Edimburg (EPDS) consiste em um instrumento de autoavaliação composto por 10 itens referentes aos sintomas depressivos frequentemente observados no puerpério ${ }^{1}$. Recentemente, em um estudo sobre as propriedades psicométricas da $\mathrm{EPDS}^{1}$, submetemos à escala 245 mulheres (média $=30,7 ; \mathrm{DP}=5,8)$ selecionadas aleatoriamente a partir dos dados de registro de internação de uma maternidade privada de Belo Horizonte-MG. As características da amostra estão descritas na Tabela 1. A Mini Plus-5.0 foi utilizada como padrão ouro para o diagnóstico de depressão. O coeficiente alfa de Cronbach de 0,87 e a área total sobre a curva ROC foi de 0,937 (erro-padrão $=0,20 ; \mathrm{p}<0,001$ ), indicando excelente capacidade da EPDS em discriminar mulheres acometidas pela DPP. O melhor ponto de corte foi o de 10 pontos, o qual apresentou $86,4 \%$ de sensibilidade e $91,1 \%$ de especificidade.

Visando explorar as propriedades psicométricas da escala, os itens do teste foram submetidos à uma análise fatorial, pela qual obtivemos os dois fatores descritos na Tabela 1. Os testes KMO $(0,896)$ e Bartlett $(\mathrm{p}<0,01)$ indicam que tal extração fatorial é adequada ${ }^{4}$. Os dois fatores, que apresentaram autovalor superior a 1, foram extraídos pelo método Maximum Likelihood, explicando juntos 49,68\% da variância. Ao final da extração, a rotação Oblimin foi aplicada. Assim como Phillips et al., o procedimento gerou um fator relacionado a sintomas depressivos e outro a sintomas de ansiedade 5 . Visando elaborar uma escala reduzida a partir da EPDS, foram escolhidos os itens com maior carga fatorial (acima de 0.6), sendo selecionados os itens 1,2 e 
Tabela 1 - Caracterização sociodemográfica da amostra

\begin{tabular}{|c|c|c|c|c|}
\hline \multicolumn{2}{|c|}{ Dados sociodemográficos } & $\begin{array}{l}\text { Depressão pós-parto } \\
(\mathrm{N}=66)\end{array}$ & $\begin{array}{l}\text { Sem depressão pós-parto } \\
\qquad(N=179)\end{array}$ & $\mathbf{p}$ \\
\hline \multicolumn{2}{|c|}{ Momento da avaliação (dias após o parto) } & $58,26 \pm 10,51$ & $58,23 \pm 9,80$ & $0,98^{*}$ \\
\hline \multicolumn{2}{|c|}{ Idade (anos) \pm DP } & $30,24 \pm 5,60$ & $30,92 \pm 5,90$ & $0,42^{* *}$ \\
\hline \multicolumn{2}{|l|}{ Número de gestações } & $1,62 \pm 0,97$ & $1,7 \pm 0,88$ & $0,53^{* * *}$ \\
\hline \multirow[t]{2}{*}{ Escolaridade } & Ensino médio & 36 & 79 & $0,14^{\dagger}$ \\
\hline & Ensino superior & 30 & 100 & \\
\hline \multirow[t]{2}{*}{ Estado civil } & Casado (ou união estável) & 53 & 142 & $0,86^{\dagger \dagger}$ \\
\hline & Solteiro & 13 & 37 & \\
\hline \multirow[t]{2}{*}{ Situação laborativa } & Exerce atividades laborais & 25 & 56 & $0,33^{t t t}$ \\
\hline & Sem atividades laborais & 41 & 123 & \\
\hline
\end{tabular}

* teste $t=0,21$; ${ }^{* *}$ teste $t=0,8 ;{ }^{* * *}$ teste $t=0,64 ;{ }^{\dagger} X^{2}=6,72 ;{ }^{t+} X^{2}=0,75 ;{ }^{\dagger+t} X^{2}=0,96$

7 (correspondentes ao fator depressão) e 3, 4 e 5 (para o fator ansiedade), os quais são sugeridos como componentes da EPDS-6. Para estes itens em conjunto o teste alfa de Cronbach teve como resultado 0,789 , um indicativo adequado de consistência interna. A área total sobre a curva ROC foi de 0,896 (erro-padrão = 0,26; $\mathrm{p}<0,001)$, mostrando que a EPDS-6 apresenta bom potencial para discriminar mulheres com DPP e sem o quadro de DPP. O melhor ponto de corte na versão reduzida foi o de 6 pontos, com sensibilidade de $81 \%$ e especificidade de $86 \%$.

Os resultados obtidos apontam que a versão reduzida da EPDS mantém suas propriedades adequadas para uso no diagnóstico da DPP. O desenvolvimento de uma versão reduzida da EPDS pode facilitar o seu uso em protocolos de rotina para investigação da saúde geral no período puerperal. Consideramos que, por

\section{Tabela 2 - Análise fatorial da EPDS}

\begin{tabular}{lcc}
\hline & \multicolumn{2}{c}{ Fator } \\
\cline { 2 - 3 } & Depressão & Ansiedade \\
\hline $\begin{array}{l}\text { 1. Eu tenho sido capaz de rir e achar } \\
\text { graça das coisas. }\end{array}$ & $\mathbf{0 , 7 5 5}$ & $-0,040$ \\
$\begin{array}{l}\text { 2. Eu sinto prazer quando penso no } \\
\text { que está por acontecer em meu dia- }\end{array}$ & $\mathbf{0 , 8 4 7}$ & $-0,124$ \\
a-dia. & & \\
$\begin{array}{l}\text { 3. Eu tenho me sentido culpada sem } \\
\text { necessidade quando as coisas saem } \\
\text { erradas. }\end{array}$ & $-0,063$ & $\mathbf{0 , 6 4 9}$ \\
$\begin{array}{l}\text { 4. Eu tenho me sentido ansiosa ou } \\
\text { preocupada sem uma boa razão. }\end{array}$ & $-0,033$ & $\mathbf{0 , 7 6 1}$ \\
$\begin{array}{l}\text { 5. Eu tenho me sentido assustada ou } \\
\text { em pânico sem um bom motivo. }\end{array}$ & $\mathbf{0 , 0 7 8}$ & $\mathbf{0 , 6 1 0}$ \\
$\begin{array}{l}\text { 6. Eu tenho me sentido esmagada } \\
\text { pelas tarefas e acontecimentos do meu } \\
\text { dia-a-dia. }\end{array}$ & 0,332 & 0,250 \\
$\begin{array}{l}\text { 7. Eu tenho me sentido tão infeliz } \\
\text { que tenho tido dificuldade de dormir. }\end{array}$ & $\mathbf{0 , 6 0 4}$ & 0,220 \\
$\begin{array}{l}\text { 8. Eu tenho me sentido triste ou } \\
\text { arrasada. }\end{array}$ & 0,555 & 0,374 \\
$\begin{array}{l}\text { 9. Eu tenho me sentido tão infeliz que } \\
\text { tenho chorado. }\end{array}$ & 0,512 & 0,367 \\
$\begin{array}{l}\text { 10. A ideia de fazer mal a mim mesma } \\
\text { passou por minha cabeça. }\end{array}$ & 0,164 & 0,412 \\
\hline
\end{tabular}

demandar menos tempo para ser aplicada, a versão reduzida da EPDS pode ser de grande valia em triagens de quadros depressivos no pós-parto, principalmente no sistema público de saúde. Estudos comparativos utilizando a EPDS e a EPDS-6 estão em andamento e poderão fornecer informações sobre as propriedades psicométricas dos dois instrumentos, suas vantagens e desvantagens.

\section{Agradecimentos}

Este trabalho recebeu financiamentos do Conselho Nacional de Desenvolvimento Científico e Tecnológico (CNPq), Fundação de Amparo à Pesquisa do Estado de Minas Gerais (FAPEMIG) e pelo Programa de Institutos Nacionais de Ciência e Tecnologia (CNPq, MCT e FAPEMIG).

Leandro Fernandes Malloy-Diniz Departamento de Psicologia, Universidade Federal de Minas Gerais (UFMG), Belo Horizonte, MG, Brasil

Programa de Pós-Graduação em Neurociências, Universidade Federal de Minas Gerais (UFMG), Belo Horizonte, MG, Brasil Instituto Nacional de Ciência e Tecnologia em Medicina Molecular (INCT-MM), Brasil

Carlos Guilherme Maciel Furtado Schlottfeldt

Departamento de Psicologia, Universidade Federal de Minas Gerais (UFMG), Belo Horizonte, MG, Brasil

Patrícia Figueira

Programa de Pós-Graduação em Neurociências, Universidade Federal de Minas Gerais (UFMG), Belo Horizonte, MG, Brasil

Fernando Silva Neves, Humberto Corrêa Programa de Pós-Graduação em Neurociências, Universidade Federal de Minas Gerais (UFMG), Belo Horizonte, MG, Brasil Departamento de Saúde Mental, Programa de Pós-Graduação em Neurociências, Universidade Federal de Minas Gerais (UFMG), Belo Horizonte, MG, Brasil Instituto Nacional de Ciência e Tecnologia em Medicina Molecular (INCT-MM), Brasil 
Financiamento e conflito de interesse

\begin{tabular}{|c|c|c|c|c|c|c|c|}
\hline $\begin{array}{c}\text { Membro do grupo } \\
\text { de autores }\end{array}$ & $\begin{array}{l}\text { Local de } \\
\text { trabalho }\end{array}$ & $\begin{array}{l}\text { Verba de } \\
\text { pesquisa }^{1}\end{array}$ & $\begin{array}{c}\text { Outro apoio à } \\
\text { pesquisa ou educação } \\
\text { médica continuada }\end{array}$ & $\begin{array}{c}\text { Honorários } \\
\text { de } \\
\text { palestrantes }\end{array}$ & $\begin{array}{l}\text { Participação } \\
\text { acionária }\end{array}$ & $\begin{array}{l}\text { Consultor/ } \\
\text { conselho } \\
\text { consultivo }\end{array}$ & Outro $^{3}$ \\
\hline $\begin{array}{l}\text { Leandro Fernandes } \\
\text { Malloy-Diniz }\end{array}$ & $\begin{array}{c}\text { UFMG } \\
\text { INCT-MM }\end{array}$ & - & - & - & - & - & - \\
\hline $\begin{array}{l}\text { Carlos Guilherme } \\
\text { Maciel Furtado } \\
\text { Schlottfeldt }\end{array}$ & UFMG & - & - & - & - & - & - \\
\hline Patrícia Figueira & UFMG & - & - & - & - & - & - \\
\hline Fernando Silva Neves & $\begin{array}{l}\text { UFMG } \\
\text { INCT-MM }\end{array}$ & - & - & - & - & - & - \\
\hline Humberto Corrêa & $\begin{array}{c}\text { UFMG } \\
\text { INCT-MM }\end{array}$ & - & - & - & - & - & - \\
\hline
\end{tabular}

* Modesto

** Significativa

*** Significativa. Montantes fornecidos à instituição do autor ou a colega onde o autor tem participação, não diretamente ao autor.

Nota: UFMG = Universidade Federal de Minas Gerais; INCT-MM = Instituto Nacional de Ciência e Tecnologia em Medicina Molecular.

Mais informações, consultar as Instruções aos Autores.

Referências

1. Figueira P, Correa H, Malloy-Diniz L, Romano-Silva MA. Edinburgh Postnatal Depression Scale for screening in the public health system. Rev Saude Publica. 2009;43(Supl 1):79-84.

2. Figueira P, Fernandes Malloy-Diniz L, Aurélio Romano-Silva M, Silva Neves F, Corrêa H. Postpartum depression and comorbid disorders: frequency and relevance to clinical management. Arch Womens Mental Health. 2009;12(6):451
3. Figueira P, Malloy-Diniz L, Campos S, Miranda DM, Romano-Silva MA, Neves FS, De Marco LA, Corrêa H. An association study between the Val66Met polymorphism 5 of the BDNF gene and postpartum depression. Arch Womens Mental Health. DOI 10.1007/s00737-010-0146-6.

4. Tabachnick BG, Fidell LS. Using multivariate statistics. Boston, MA: Allyn and Bacon; 2001

5. Phillips J, Sharpe L, Matthey S. Validation of the subscales of the Edinburgh Postnatal Depression Scale in a sample of women with unsettled infants. J Affect Disord. 2009;118(1-3):101-12. 